\title{
Pulse Phase Thermography: IMPACT DAMAge Retrieval
}

\section{Petra Bagavac $^{1}$, Lovre Krstulović-Opara ${ }^{1}$, Željko Domazet ${ }^{1}$}

1 University of Split, Faculty of Electrical engineering, Mechanical Engineering and Naval

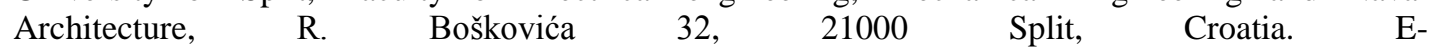
mail: petra.bagavac@fesb.hr, lovre.krstulovic-opara@fesb.hr, zeljko.domazet@fesb.hr.

\section{Introduction}

Impact damage in composite materials is hard to reveal with methods of IR thermography as flaw has no sharp edges.

Generally authors [1-4] are inspecting composite materials which have been deliberately damaged with flat bottom holes or with inserts of foreign material (e. g. Teflon) causing sharp images. Authors in $[5,6]$ analyzed impacted specimens with methods of passive thermography during the impact, where IR camera recorded increase in temperature caused by the release of heat energy liberated by the plastic deformation.

\section{The Pulse-Phase Thermography}

A typical configuration to perform a PPT is shown on Fig. 1. Specimen is heated with high power photographic flash and temperature decay on the surface of the specimen is recorded with IR camera. After acquisition, $\mathrm{N}$ thermograms form the sequence written as 3D array, where $x$ and $y$ are pixel position and $z$ is time ax $[7,8]$.

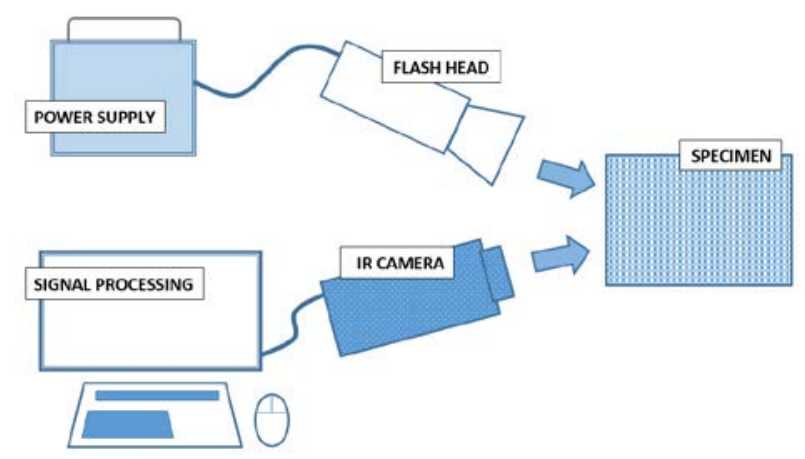

Fig. 1. PPT experimental setup.

Follows, temperature decay of observed pixel $(i, j)$ is a vector containing temperature values during observed time ax. Temperature decay curve of each pixel is reconstructed by applying the Fourier Transform. By using the FT is possible to reconstruct any $1 \mathrm{D}$ function as a sum of sinusoidal terms of increasing frequency.

$$
F_{n}=\Delta t \sum_{k=0}^{N-1} T(k \Delta t)^{-j 2 \pi \omega k / N}=R e_{n}+I m_{n}
$$

where $j^{2}=-1 ; n$ designates the frequency increment $(n=0,1, \ldots, N) ; \Delta t$ is the sampling interval; $R e$ and $I m$ are the real and the imagniray parts of the transform, respectively [8]. Real and imaginary parts of Eq. (1) can be used to calculate the amplitude and the phase of the transform in order to reconstruct 3D matrices as illustrated in Fig. 2.
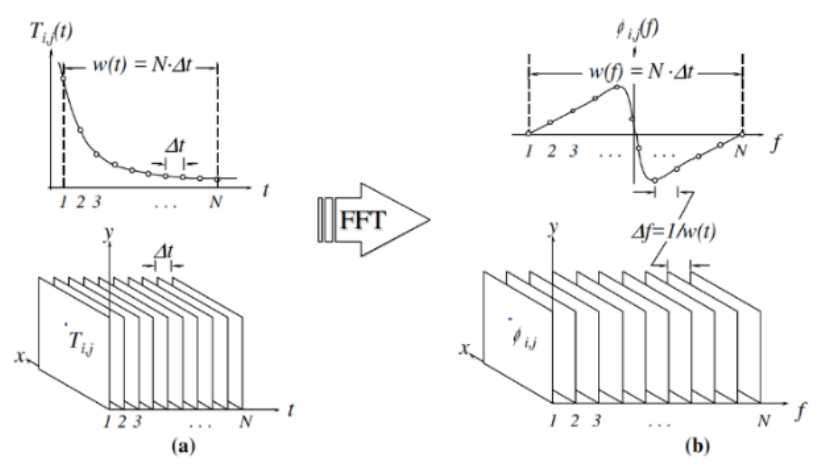

Fig. 2. (a) Thermogram sequence and temperature profile for a pixel, and (b) phasegram sequence after application of the FFT.

\section{Experimental procedure and results}

Test specimens are made from the GFRP (specimen $A$ ) and the CFRP (specimen $B$ ). Specimens are deliberately damaged with the controlled metal rod impactor. The metal rod was released from the height of $2 \mathrm{~m}$, releasing $19.62 \mathrm{~J}$ of impact energy.

The surface of the specimen is heated by the Hensel $^{\circledR}$ flash head and power supply, able to emit $6 \mathrm{~kJ}$ in $1 / 400 \mathrm{~s}$. Measurements of exposed specimens are taken with cooled MW FLIR ${ }^{\circledR}$ SC 5000 Infrared camera. Resulting thermogram sequence for each specimen is shown in Fig. 3.

The resulting thermographic sequence is processed by the $M A T L A B{ }^{\circledR}$ program package, and resulting PPT phasegrams are shown in Fig. 4. 

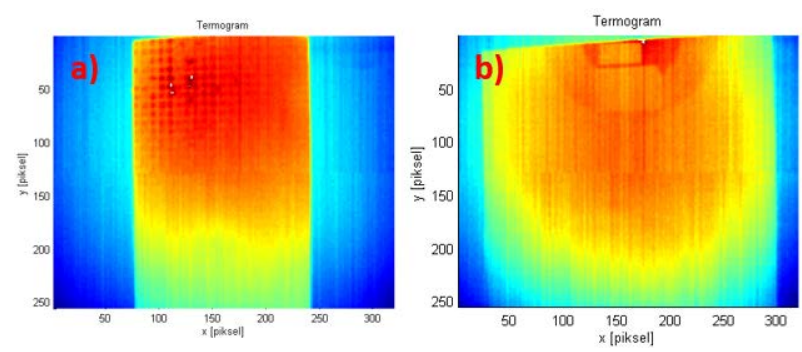

Fig. 3. Raw data thermogram: a) GFRP, b) CFRP.
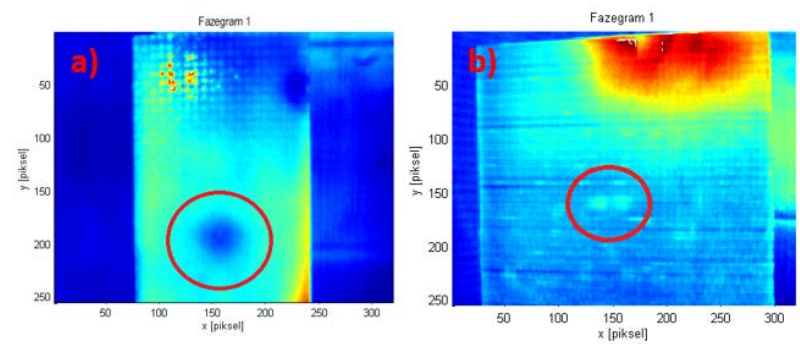

Fig. 4. Phasegram: a) GFRP, b) CFRP.

\section{Preprocessing of the row data}

Thermal decays are characterized by the high level of noise resulting from differences in the responsivity of detectors to the incoming irradiance. It is a common problem of focal plane arrays (FPA) detectors. In this work in order to reduce the noise level, the preprocessing technique based on fitting the decay curves with smooth polynomial curve, is proposed. The thermal decay for each pixel of specimen $A$ is fitted by the polynomial curve $T(t)=a t^{2}+b t+c$ (Fig. 5a). The thermal decay for each pixel of specimen $\mathrm{B}$ is fitted with the exponential curve $T(t)=a e^{b t}+$ $c e^{d t}$, (Fig. 5b).
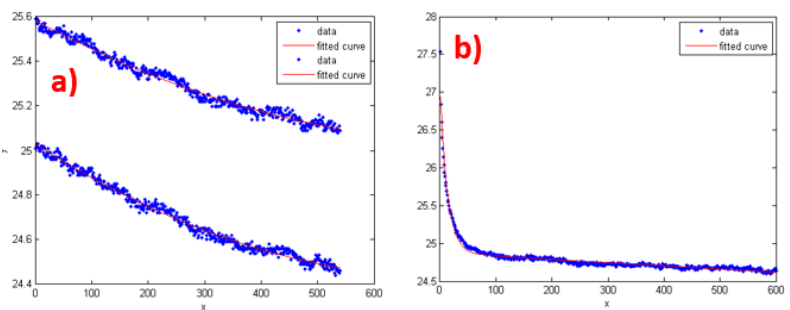

Fig. 5. Raw data and fitted curve: a) GFRP, b) CFRP.
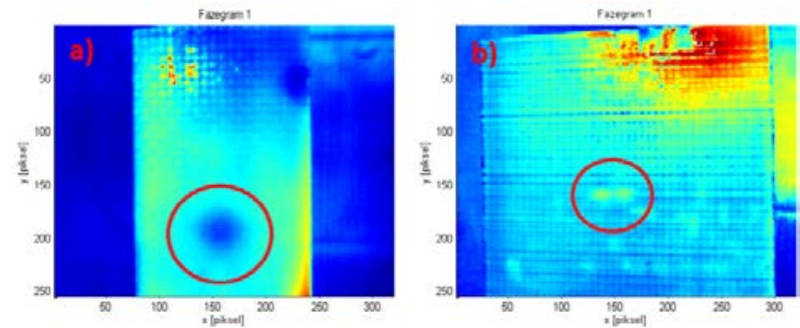

Fig. 6. Fitted data phasegram: a) GFRP, b) CFRP.
After fitting the row data, fitted data are reconstructed by the Fourier Transform and phase images are shown in Fig. 6. The increased detail of impact damage for CFRP specimen is shown in Figure 7.
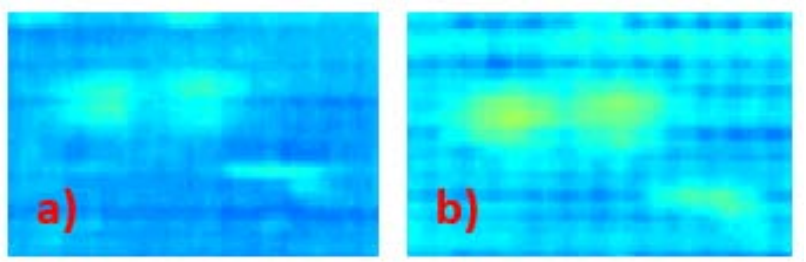

Fig. 7. Impact damage phasegram for CFRP sample: a) before preprocessing, b) after preprocessing.

\section{Concluding remarks}

The progressive and continuous increase in computer power together with the availability of transformation tools in used software packages encourages further developments in the field.

\section{References}

[1] Ibarra-Castanedo, C.: „Quantitative subsurface defect evaluation by pulsed phase termography: depth retrieval with phase“, Ph. D. Thesis, Laval University, 2005

[2] C. Meola, G. M. Carlomagno, L. Giorleo: „Geometrical Limitations to Detection of Defects in Composites by Means of Infrared Thermography“, Journal of Nondestructive evaluation, 2004

[3] Mohammed A. Omar, Yi Zhou: “A quantitive review of three thermography processing routines”, Infrared Physics \& Technology, p. 300306, 2008

[4] Takahide Sakagami, Shiro Kubo: “Applications of pulse thermography and lock in thermography to quantitative nondestructive evaluations”, Infrared Physics \& Technology, p. 211-218, 2002

[5] Meola, C.; Carlomagno, G. M.; Ricci, F.; Lopresto, V.; Caprino, G.: "Investigation of Impact Damage in Composites with Infrared Thermography”, $6^{\text {th }}$ NDT in Progress, Prague, 2011

[6] C. Ibarra Castanedo, A. Bendada, X. Maldague: “Themographic Image for NDT”, IV Conferencia Panamericana de END, 2007

[7] C. Ibarra-Castanedo, X. Maldague: “Defect Depth Retrieval from Pulsed Phase Thermographic Data on Plexiglas and Aluminium Samples”, Proceedings of SPIE, 2004

[8] Maldaque X. P. and Marinetti S.: "Phulse phase infrared thermography”, J. Appl. Phys., p. 26942698, 1996 